\title{
Structure in the classification of stimuli differing on several continuous attributes'
}

\author{
HILDA WING ${ }^{2}$ AND WILLIAM BEVAN \\ THE JOHNS HOPKINS UNIVERSITY
}

Subjects were requested to sort sets of multidimensional stimuli differing in tint or shade, size, shape, and orientation. Each $S$ received either 64 triangles selected at random from a larger population or 16 selected at random from the sample of 64. On one sort he was allowed to select the number of categories to be used; otherwise, he was required to distribute the stimuli among four, five, and six categories, respectively. The predominant strategy proved to be simple abstraction, with incomplete generalization a frequent alternative. On all but a few of the sorts, the sorting principle involved the use of one or a pair of attributes. In addition, $S$ s displayed a marked tendency toward numerical balance in their sorts and, when allowed to select the number of categories to be used, to limit them to a relatively small number. Imai has previously reported numerical balance and the use of a limited number of categories also to characterize the sorting of stimuli differing on discontinuous attributes. Informational measures proved helpful in describing such aspects of classification behavior as response uncertainty, numerical balance, and efficiency in abstraction.

Scholars have had a long-standing interest in how man perceptually organizes the world that surrounds him. The contrasting traditions of empiricistic associationism and nativistic Gestalt theory have at least one thing in common: They are referenced to the properties of the ambient physical stimulus array. But recent studies of classification behavior indicate that other variables also play a significant role in the ordering of stimuli. Imai (1966), for example, has shown that subjects display a strong tendency to arrange stimuli into equal-sized sets (numerical balance) and Imai and Garner (1968) report that the structure of stimulus populations has a greater influence upon classification than simple similarity and difference relations among members of a sample.

This study extends the work of Imai and Garner in that it deals with the classification of stimuli that vary on attributes of a continuous nature and involves the classification of different size samples from the same stimulus population.

\section{Subjects}

Six men and nine women were paid volunteers from undergraduate classes of the Johns Hopkins 1967 Summer Session. Each was tested individually in a single session of approximately $1 \cdot \mathrm{h}$ duration.

\section{Stimuli}

The stimuli were blue isosceles triangles each cut from Color Aid paper and mounted at the center of a 5-in. square white card. A thin strip of black tape near the bottom edge of each card indicated the orientation of the stimulus form. Clear acetate on the front prevented damage to the cards and minimized local cues.

The attributes on which the triangles varied were color (chromatic tint or shade), shape, size, and figural orientation. There were the eight blues of the Color Aid series, varying between Munsell Color $\mathrm{PB} / \mathrm{V} 3 / \mathrm{C} 3$ and $\mathrm{PB} / \mathrm{V} 7 / \mathrm{C} 6$. The several shapes had height-to-base ratios of $1: 2,1.33: 1.50 ; 1.50: 1.33$; and $2: 1$. Sizes were $.50, .75,1.00$, or $1.25 \mathrm{sq}$ in. and the apex of the individual figures was oriented toward 0 (East), 45, 90, 135, 180, 225,270 , or $315 \mathrm{deg}$. All possible combinations of these stimulus values produced a population of 1024 unique forms.

Two sets drawn from this population constituted the stimulus samples used in the present study. The larger sample of 64 members was selected at random, without replacement. The smaller, of 16 members, was selected at random from the 64-member sample, again without replacement.

\section{Experimental Design}

The Ss were divided into five groups of three Ss each. Groups 1 and 3 consisted of males, 2, 4, and 5 of females. All were required to sort both large and small samples and to make both unrestricted and restricted (4-, 5-, and 6-class) sorts. Groups 1, 2, and 5 sorted the small set before the large set; for Groups 3 and 4 , this order was reversed. Groups 1 through 4 made unrestricted sorts, followed by 4-, 5-, and 6-class restricted sorts. Group 5 made an unrestricted sort followed by 6-, 5-, and 4-class restricted sorts.

\section{Procedure}

$S$ was seated at a large table opposite $E$ and told that he would be asked to sort a set of simple forms into several piles. $E$ explained that there were various possible ways of doing this but that she was interested only in the way that $S$ thought was most appropriate. It was also noted that he could take as much time as he wished, within reason, to make his sort. A set of cards was then spread in random array on the table and $S$ was asked to make his first sort. When this sort was completed, E picked up the piles individually for later recording, spread out a fresh set of stimuli, and called for the next sort. This procedure was repeated until all required sorts had been made. While $S$ made his sorts, $E$ made observations of his sorting behavior and, before she excused him, asked him to describe the sorting principle or principles he had employed.

\section{Information Measures}

A variety of measures of uncertainty were computed from each sort.

(a) $U_{r}$ is the uncertainty of the distribution of stimuli as sorted by $S$. It increases with increases in the number of classes formed in a sort. Secondarily, it reflects the extent to which the principle of numerical balance has guided the sort, since, if the number of stimuli is the same for all classes, $U_{r}$ is maximal.

(b) The ratio, $U_{r} / \operatorname{Max} U_{r}$, measures the prevalence of numerical balance in the sorting behavior, and is, at the same time, a measure that is logically free of the effect both of number of classes and size of sample. A value of 1.00 indicates the same number of stimuli in each class; a smaller ratio, the existence of unequal-size classes.

(c) $I_{t}$ indicates the amount of information transmitted in the classification when a sort is determined by a single attribute or by attributes functioning in combination. A total of $14 I_{t}$ values, representing all attributes and attribute combinations, was 
computed for each sort in the present experiment. This computation is analogous to partitioning the variance in an analysis of variance design. For this metric, however, only those values associated with single attributes are independent, since the $I_{t_{i}}$ scores for combinations include simple effects as well as interactions.

(d) Because the stimulus sets sorted were samples drawn from the same population, their attribute structure is redundant. Thus, when sorting conforms to one criterion (that is, occurs in terms of a particular attribute or combination of attributes), the information transmitted by each of the other possible sorting criteria $\left(\mathrm{I}_{t_{j}}\right)$ will not be minimal. To correct the measures of transmitted information for this redundancy, a ratio score, $I_{t_{i} / \text { Max }} I_{t_{i}}$ was useful. This measure indicates the discrepancy between the information available in an attribute or combination of attributes and the amount actually used in the sort and thus reflects the extent to which a particular sorting criterion is applied by $S$ in producing the sort. A ratio of 1.00 means that $S$ is discriminating as many differences among stimuli on a particular attribute or combination of attributes as actually exist in the sample. As in the case of response uncertainty, the use of the measure in ratio form makes possible compensation for differences in numbers of classes and the sample size involved. 3

(e) Another ratio, $U_{r_{i}} / U_{I}$, may be used to indicate the relative prominence of particular attributes or combinations of attributes in determining a sort. This measure is less useful, however, since it is uncorrected for sample redundancy.

\section{The Evaluation of Sorting Behavior}

Characterizations of $S$ behavior resulted from two types of evaluation: examination of the information measures and a qualitative analysis of the posttest protocols.

The $U_{r_{i}} / U_{r}$ ratios for each stimulus attribute, considered singly, were examined for homogeneity. The criterial attribute (i.e., the one upon which sorting was based) was then identified. To be criterial for any particular sort, an attribute either must have achieved a ratio of .75 or one at least $20 \%$ larger than any other. The $I_{t_{i}} /$ Max $I_{t_{i}}$ scores were then examined to check the consistency of this classification. If no single attribute was dominant, ratios were reviewed for attributes operating in pairs, or in combinations of three. In a total of 120 sorts, 108 were judged to have been based on one or two attributes.

Inspection of the protocols suggested a variety of conceptual strategies or principles for sorting. Virtually all were of two general abstractive types: orthogonal and nonorthogonal.

(A) Orthogonal strategies

(1) Simple abstraction. S sorted in terms of one attribute, ignoring the remaining three.

(2) Partial abstraction. S sorted in terms of two attributes, ignoring the remaining two.

(3) Modified partial abstraction. S sorted on the basis of two attributes, but the values of the secondary attribute were not the same for all values of the primary attribute.

(B) Nonorthogonal strategies

(1) Incomplete generalization. S sorted according to one attribute, but subdivided one of the resulting classes in terms of values on a second attribute.

(2) Chaining. S sorted according to one attribute, but subdivided one or more of the resulting classes in terms of values on a second attribute, while the remaining classes were subdivided in terms of a third.

(C) Miscellaneous strategies. Strategies that could not be assigned one of the above types.

\section{Statistical Treatment of the Information Measures}

Each of the information measures was analyzed in a multifactor design, with repeated measures. When a single score represented each sort, the variables of sample size and type of sort were assessed for each $\mathrm{S}$, repeated across groups. The ratio scores were subjected to the arc sine transformation following the suggestion of Winer (1962, p. 221), in order to insure their normality.

\section{RESULTS AND DISCUSSION}

Sorting Strategy, Sorting Criterion, Sample Size, and Sorting Instructions

Table 1 summarizes the frequency with which the several sorting strategies were used and the way in which they relate to criterial attributes or attribute combinations. Table 2 relates the frequency of strategy usage to the size of the stimulus sample and the number of categories specified by the $E$. Inspection of these tables indicates, first of all, an overwhelming preference for a relatively simple strategy. Simple abstraction was employed on 48 of the sorts, or $40 \%$ of the time, and incomple te generalization, on 32 of the sorts or approximately $27 \%$ of the time. Of the two

Table 1

The Frequency with which the Several Sorting Strategies were used, Identified in Terms of the Attribute or Combination of Attributes Involved as the Sorting Criterion

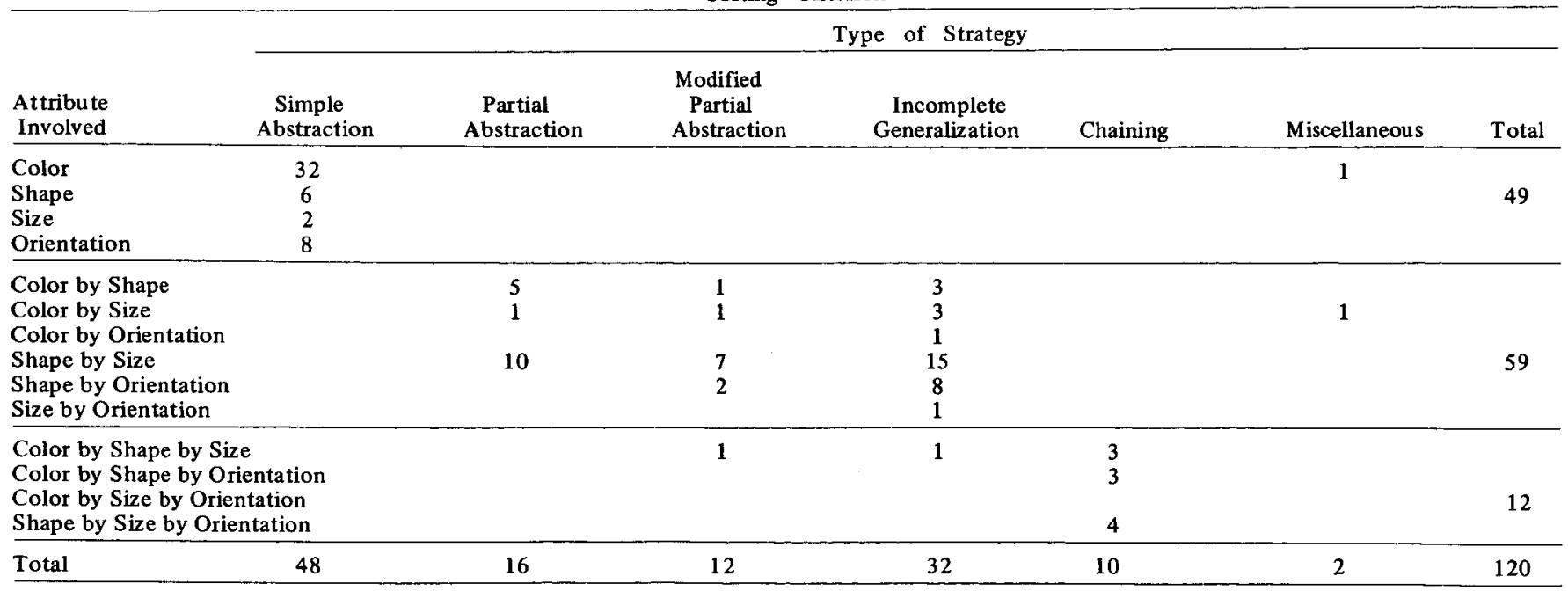


Table 2

The Frequency with which the Several Sorting Strategies were used, Identified in Terms of Sample Size and the Number of Categories Requested

\begin{tabular}{|c|c|c|c|c|c|c|c|c|}
\hline \multirow{2}{*}{$\begin{array}{l}\text { Sample } \\
\text { Size }\end{array}$} & \multirow{2}{*}{$\begin{array}{l}\text { Categories } \\
\text { Requested }\end{array}$} & \multicolumn{7}{|c|}{ Type of Strategy } \\
\hline & & $\begin{array}{c}\text { Simple } \\
\text { Abstraction }\end{array}$ & $\begin{array}{c}\text { Partial } \\
\text { Abstraction }\end{array}$ & $\begin{array}{c}\text { Modified } \\
\text { Partial } \\
\text { Abstraction }\end{array}$ & $\begin{array}{c}\text { Incomplete } \\
\text { Generalization }\end{array}$ & Chaining & Miscellaneous & Total \\
\hline 16 & $\begin{array}{l}\text { Unspecified } \\
4 \\
5 \\
6 \\
\end{array}$ & $\begin{array}{l}8 \\
8 \\
4 \\
2\end{array}$ & $\begin{array}{l}2 \\
1 \\
1 \\
7\end{array}$ & $\begin{array}{l}4 \\
1 \\
1\end{array}$ & $\begin{array}{l}1 \\
4 \\
7 \\
2\end{array}$ & $\begin{array}{l}1 \\
1 \\
3 \\
\end{array}$ & $\begin{array}{l}1 \\
1 \\
\end{array}$ & 60 \\
\hline 64 & $\begin{array}{c}\text { Unspecified } \\
4 \\
5 \\
6 \\
\end{array}$ & $\begin{array}{l}8 \\
9 \\
6 \\
3\end{array}$ & $\begin{array}{l}1 \\
2 \\
2\end{array}$ & $\begin{array}{l}3 \\
1 \\
2\end{array}$ & $\begin{array}{l}2 \\
3 \\
6 \\
7\end{array}$ & $\begin{array}{l}1 \\
1 \\
2 \\
1\end{array}$ & & 60 \\
\hline Total & & 48 & 16 & 12 & 32 & 10 & 2 & 120 \\
\hline
\end{tabular}

major classes of strategies used, the orthogonal or nonorthogonal, the orthogonal was clearly more frequently preferred: $76 \mathrm{vs} 44$ $\left(\chi^{2}=14.07, \mathrm{df}=3, \mathrm{p}<.01\right)$. Table 1 indicates that $\mathrm{Ss}$, overall, used a single attribute or a pair of attributes with roughly comparable frequency ( $41 \%$ vs about $50 \%$ of the time). A combination of three at tributes was used relatively infrequently, i.e., only about $9 \%$ of the time. When a single attribute was used, color was the overwhelming favorite (selected 32 out of 49 times). When a pair was used, the most likely combination was shape and size (selected 32 out of 59 times). This may follow from the fact that in the present design color was represented by eight levels while size and shape were each represented by four. Further work may demonstrate that the perceived number of levels per attribute influences the strategy selected for a given task requirement. There was also a clear relationship between the type of strategy employed and the type of criterion selected. The strategy of simple abstraction involves use of a single attribute as criterion. Incomplete generalization involves use of a pair of attributes as criterion. Chaining involves a combination of three attributes as the sorting criterion. By far the most popular approach to sorting in this study was to sort for color, ignoring all other considerations. When partial generalization was used, the most frequently adopted criterion involved combining shape and size.

Inspection of Table 2 yields no overwhelming impression of a dependence of strategy employed upon number of categories used, with the possible exception that simple abstraction appears more likely to have involved fewer categories and incomplete generalization more. When sample size was small, orthogonal strategies were used more frequently than nonorthogonal $\left(\chi^{2}=9.60, \mathrm{df}=3, \mathrm{p}<.01\right)$, but this did not prove to be the case when the sample size was large $\left(\chi^{2}=4.72, \mathrm{df}=3, \mathrm{p}>.05\right)$.

Imai (1966) has suggested that incomplete generalization is employed when the structure of the stimulus set is incompatible with the number of categories requested and it is of interest that, in the present experiment, none of the stimulus attributes could be evenly sorted into five or six categories. Chaining was used infrequently and, as noted, always with a complex criterion. While the resulting sorting behavior is also complex it may reflect a relatively primitive cognitive condition. Consistent with this inference is Piaget and Inhelder's observation (1964, cf. Chap. 2) of a similar strategy in young children.

\section{Uncertainty in the Sorting Responses}

Table 3 summarizes $U_{r}$ as a function of the type of instructions presented the $S$ (i.e., the number of categories used in sorting) and the number of stimuli to be sorted. The usual increase in uncertainty with increased number of classes is evident [F(category) $(3,30)=14.45, \mathrm{p}<.001]$. Curiously, however, increasing the size of the sample to be sorted had no effect upon $\mathrm{U}_{\mathrm{r}} ; \mathrm{F}($ size $)(1,10)=0.88, \mathrm{p}>.05$. Indeed, even when the $\mathrm{S}$ was free to select the number of categories to be used, he consistently chose a relatively small number regardless of sample size; on the average, 4.2 with the 16-stimulus sample and 5.3 with the 64 . Since the larger sample necessarily contained more information, this tendency to employ a small number of categories, also observed by Imai (1966), is another reflection of the predominance of the abstractive strategy.

\section{The Efficiency of Sorting Behavior}

As noted earlier, the ratio, $\mathrm{I}_{\mathrm{t}_{\mathrm{i}}} / \mathrm{Max} \mathrm{I}_{\mathrm{t}_{\mathrm{i}}}$, indicates the relative amount of the information, from that available for a particular attribute or attribute combination, actually used in sorting to that criterion. Inspection of Fig. 1 indicates that efficiency of classification varied widely depending on the criterion adopted and the particular conditions of sorting. Under the conditions of best performance, Ss at best appeared to utilize only about $80 \%$ of the available information while under another condition, they ignored as much as $90 \%$ of the information available.

Certain general ielationships among the data seem clear. Performance appears to have been better for the 16-stimulus sample than for the 64 , although the latter had 1.5 times as much information potentially available for transmission as the former. The average efficiency level for the sorts made with the small sample was .54 while that for the large was .34 . More information tended to be transmitted when a larger number, as compared to a smaller number, of categories was involved. When four categories were specified, an average of $37 \%$ of the available information was transmitted; when six were called for, $49 \%$ was used. Color and size appeared generally to be more effective criteria for sorting than were shape and orientation (.45 and .41 vs .39 and .33). When single attributes were involved, efficiency averaged .39; when a combination of two was used, .45 ; and with three, .46 . In addition, Fig. 1 suggests that as the number of attributes included in the sorting strategy increased, the differences in performance identified with different strategies and different sorting requirements were reduced.

Table 3

Response Uncertainty in Bits as a Function of Stimulus Sample Size and Sorting Instructions

\begin{tabular}{ccc}
\hline & \multicolumn{3}{c}{ Sample Size } \\
\cline { 2 - 3 } No. of Categories Used & 16 & 64 \\
\hline 4 & 1.89 & 1.86 \\
Unspecified (4.2) & 2.07 & - \\
5 & 2.22 & 2.20 \\
Unspecified (5.3) & - & 2.44 \\
6 & 2.49 & 2.40 \\
\hline
\end{tabular}



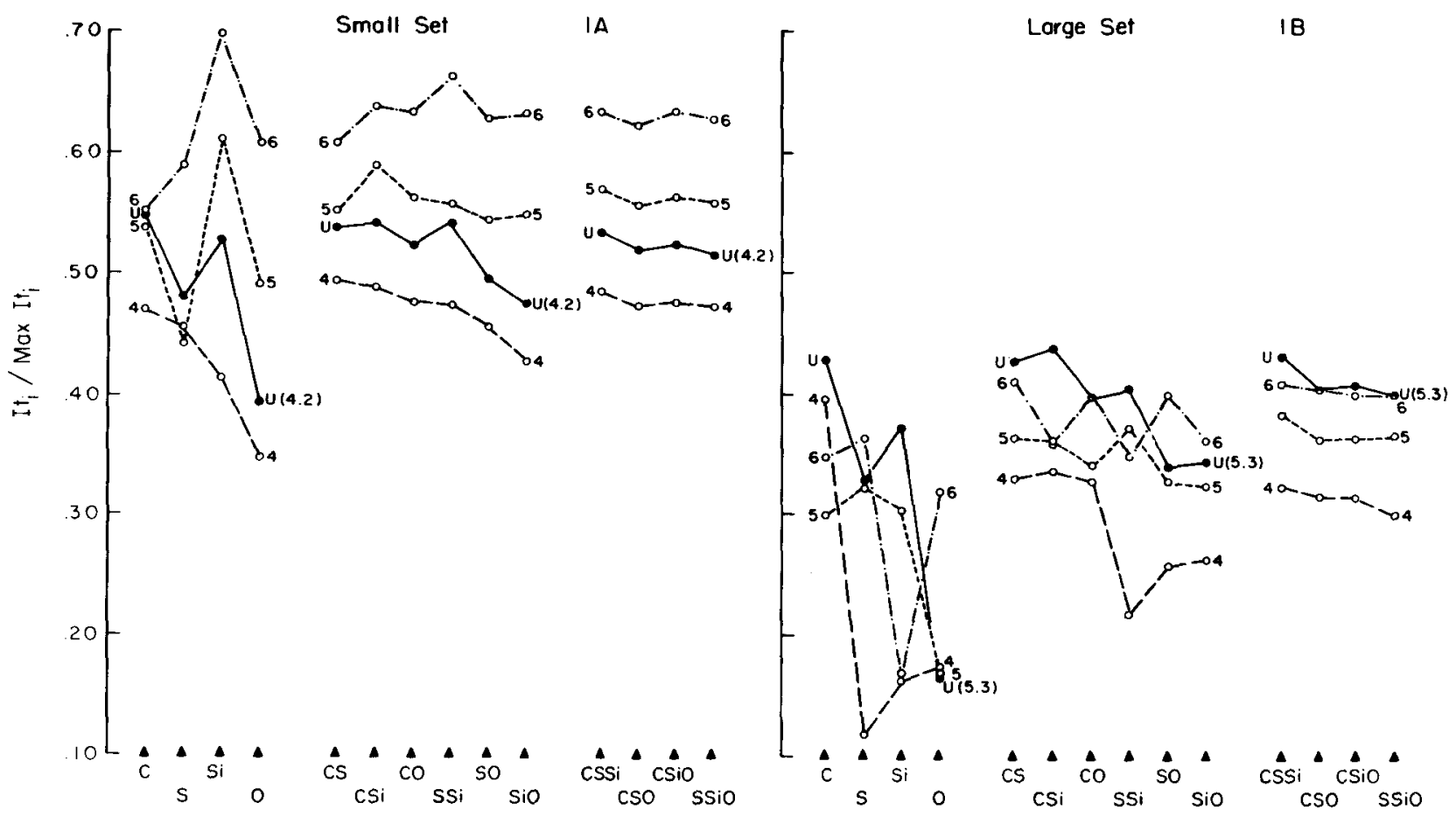

Ratio of Information Transmitted per Aftribute to Maximum Information Available per Attribute (It $/$ Max $\mathrm{It}_{\mathrm{i}}$ )

Fig. 1. Efficiency of sorting behavior as a function of the attribute or combination of attributes involved and the size of the stimulus sample available to the S. Fig. $1 \mathrm{~A}$ presents data for the 16-stimulus sample, Fig. $1 \mathrm{~B}$ for the 64. The attributes are coded as follows: $\mathrm{C}$ indicates color; $\mathrm{S}$, shape; $\mathrm{Si}$, size; and $\mathrm{O}$, orientation. The numerical labels applied to the curves indicate the number of categories employed.

However, a word of caution is in order. The scores associated with sorting based on combinations of two or more attributes represent, as pointed out earlier, the independent influence of each component, plus the interaction or interactions, and must be interpreted with great care. Accordingly, statistical evaluation was carried out upon the data obtained for single attributes only. Under these circumstances, all F-ratios were consistent with expectation: $F($ size $) \quad(1,10)=269.82, \quad p<.001 ; \quad F$ (category) $(3,30)=13.83, \mathrm{p}<.001 ; \mathrm{F}$ (attribute) $(3,30)=2.60, \mathrm{p}>.05 ;$ $\mathrm{F}$ (size by category) $(3,30)=4.71, \mathrm{p}<.01 ; \mathrm{F}$ (size by attribute) $(3,30)=2.89, p>.05 ; F($ category by attribute $)(9,90)=1.43$, $\mathrm{p}>.05 ; \mathrm{F}$ (size by category by attribute) $(9,90)=1.65, \mathrm{p}>.05$.

\section{The Tendency Toward Numerical Balance}

A recent memory experiment of Broadbent and Gregory, cited by Broadbent (1966), indicates quite clearly that performance may be influenced markedly by criteria for classifying stimuli that are completely irrelevant as far as the formal requirements of the task are concerned. Imai's observation (1966) of the tendency of Ss to arrange stimuli in equal-sized categories constitutes another example of the same phenomenon.

Table 4

The Tendency Toward Numberical Balance as Indicated by $U_{r} / M a x \quad U_{r}$

\begin{tabular}{ccc} 
& \multicolumn{3}{c}{ Sample Size } \\
\cline { 2 - 3 } No. of Categories Used & 16 & 64 \\
\hline 4 & .94 & .93 \\
Unspecified $(4.2)$ & .97 & - \\
5 & .96 & .95 \\
Unspecified $(5.3)$ & -96 & .96 \\
6 & .96 & .93 \\
\hline
\end{tabular}

The strategy of numerical balance was a predominant one in the present experiment. Table 4 presents $U_{r} / M a x U_{r}$ ratios averaged across attributes and attribute combinations and summarized, for convenience, in terms of the number of categories employed and the number of stimuli sorted. A ratio of 1.00 indicates that all categories in a given sort are of the same numerical size. Two things are immediately apparent from Table 4: (1) There was a strong tendency toward numerical balance; and (2) numerical balance, as a strategy, was uninfluenced by either sample size or number of required categories. An analysis of variance of the $U_{r} / M a x U_{r}$ data yielded no significant $F$ s.

\section{Consistency in the Application of Strategies}

An examination of the content of individual sorts revealed Ss to be strikingly consistent in the use of classification strategies. After the strategy applied in each sort had been inferred by $E$, a count was made of the deviations in its application. The occurrence of inconsistencies proved to be markedly low. In the case of the 16-stimulus sample, there were no errors in half the sorts, only one error in another $35 \%$, and no sort contained more than five errors. With the 64-stimulus set, a third of the set contained zero or one errors and half had three or less. E also compared her inference of the strategy involved in each sort with that which S said he had used. Discrepancies occurred on 26 of the 117 scorable protocols. At least one discrepancy was found for each S, two for three Ss, four for one, and six for another. Twelve of the 26 discrepancies involved a confusion between shape and a combination of shape and size as criteria. Nine others similarly involved the identification of a single attribute when a combination was used while in four instances, the opposite confusion occurred. 
REFERENCES

BROADBENT, D. E. The well ordered mind. American Education Research Journal, 1966, 3, 281-295.

IMAI, S. Classification of sets of stimuli with different stimulus characteristics and numerical properties. Perception \& Psychophysics, $1966,1,48-54$.

IMAI, S., \& GARNER, W. R. Structure in perceptual classification. Psychonomic Monograph Supplements, 1968, 2, No. 9 (Whole No. 25), 153-172.

INHELDER, B., \& PIAGET, J. The early growth of logic in the child. New York: Harper and Row, 1964.

WINER, B. J. Statistical principles in experimental design. New York: McGraw-Hill, 1962.

\section{NOTES}

1. This experiment was performed under Contract N00014-67-A-01630001 between The Johns Hopkins University and the Physiological Psychology Branch, Office of Naval Research.

2. Address: Department of Psychology, The Johns Hopkins University, Baltimore, Maryland 21218.

3. Thanks are due Mr. A. G. Carlton, Principal Scientist, Applied Phy sics Laboratory of The Johns Hopkins University for suggesting the abstraction measure.

(Accepted for publication January 23, 1969.) 